\title{
A new paradigm shift: from smooth to rough to hybrid implants - Opinion paper
}

\author{
Vaartjes $\mathrm{JW}^{1}$ \\ Affiliations: \\ 1. De Kliniek voor tandheelkunde (private \\ practice) \\ Utrecht, Netherlands

\section{Corresponding author:} \\ Jan Willem Vaartjes \\ vaartjes@tandarts.nl
}

\section{ABSTRACT}

Implant therapy provides his survival rates while on the other hand the prevalence of peri-implantitis became a concern in the last 20 years. With the lack of enough longterm (>20 years) studies it's still uncertain if the high survival rates achieved with the smooth Bränemark implants are transferable to the modern rough implants we use nowadays. Risk factors for peri-implantitis change over time in our patient groups and rough surfaces will be likely be exposed to the oral environment at a certain moment. There are alarming indications that exposed rough implants are more prone to periimplantitis than smooth implants. Also treating peri-implantitis on a rough surface has been found much less predictable. This knowledge is used by different companies to develop a new group of implant surfaces, called hybrid implants. Implants with an apical rough surface for optimal bone to implant contact and short healing periods and a smooth coronal parts for a safe soft tissue integration in case it ever will be exposed to the oral environment. In this article cases are shown that with hybrid implants complex cases can be treated with aesthetic outcomes. It is the start of a whole new era of implant surface development.

\section{KEYWORDS}

Implant surface, hybrid, surface, machined, smooth, long-term success, periimplantitis, implant 
Implant therapy is one of the most successful disciplines in dentistry. It has given us so many extra possibilities in the last decades and millions of patients were helped who had otherwise been dental cripples. Scientific research mainly consisting out of studies with 1 to 10 years observation periods are regularly published with high survival rates. On the other hand, alarming reports about peri-implantitis are popping up more and more frequently in our society, even to a point that questions were asked in the British parliament. Baroness Gardner of Parkes said that peri-implantitis was an important and growing health problem and 'there needs to be an awareness and a degree of understanding of the present position and the growing risks associated with this increasingly popular form of dental treatment'. At the same time in Sweden studies were published with a high prevalence of peri-implantitis and a well-known population-based study ${ }^{1}$ about the effectiveness of implant therapy stated that within 9 years 1 out of 13 patients had lost at least 1 implant. With this survival rate we might not be competitive anymore compared to alternative treatments as conventional bridgework. Besides the survival rate, also the extra measures, morbidity and costs involved for treating implant complications should be considered. Since peri-implantitis is only treatable with an extensive debridement and open flap procedure, it is often a surgical procedure with more co-morbidity then the initial implant placement and has an uncertain outcome.

What lies behind the fact that the literature produces these high survival rates while on the other hand questions are raised about the grown prevalence of peri-implantitis? In the year 2005 there were only 19 studies published about peri-implantitis while from 2011 the yearly amount of publications exploded to 279 (Figure 1). Common explanations are the more widespread use of implants and the less strict indication for the treatment itself. However, we should not ignore the fact that during the early 00's smooth implants were fully replaced by (moderate) rough implants. Since peri-implantitis at least takes years to develop, the trend we see in the literature is what could be expected when hypothesizing that rough implants are more prone to periimplantitis than smooth implants.

The possible reason why this effect is not clearly seen in the prospective randomized trials, is next to the short follow up periods also the fact that only patients with strong inclusion criteria and highly skilled professionals are part of the programs. For example, patients with poor oral hygiene, (mild) periodontitis, diabetes, other systemic diseases or smoking are often excluded. Above this the group patients undergo a thorough follow up and are included in extensive prophylactic after care programs. These criteria are often not corresponding with the average patient in our daily office. We sometimes have to make exceptions and patients can also drop out of our regular maintenance schedules. Besides this, risk factors change over time. Our group of implant patients will eventually become old which has a dramatic effect on oral hygiene levels, the presence of systemic diseases and use of different medicines. All these factors have a negative influence on oral health and the resistance to infection around teeth and specially the already more vulnerable implants of these patients. A patient who was first a suitable implant candidate could easily be 20 years later a patient in which no one would consider placing an implant. There are only a few implant studies which has the essential follow up period of more than 20 years. And these studies are merely conducted with smooth (machined surface) implants.

A group of clinicians and scientists in Europe (eg. Prof Massimo Simion and Dr. Philippe Khayat) used the proven long-term stability of smooth implants to develop a new group of implant surfaces. Implants with an apical rough surface for optimal bone to implant contact and short healing periods and a smooth coronal parts for a safe soft tissue integration in case when it ever will be exposed to the oral environment. This was the start of a paradigm shift around what type of surface really is needed on which part of the implant.

Although the general opinion is that the rough surface of an implant should not be exposed to the oral cavity, it is often neglected that this exposition is very likely to happen at some point in every implant. This due to bone remodeling around the neck of an implant, horizontal and vertical resorption of the jaws and removal of neighboring teeth. Derks discovered in the large population study in Sweden ${ }^{1}$ that almost every implant regardless of brand and type has at least an estimated bone loss of $2 \mathrm{~mm}$ after already 10 years. A period which should be considered just a minor part of the lifetime an implant. It is expected that with this $2 \mathrm{~mm}$ bone loss most of these implants will have its rough surface exposed to the oral environment. This will not only happen over time, but also in the first year. We all experience a small amount of problems in the initial phase after surgery, like wound infections, failed GBR procedures and minor complications as cover screw exposure for example caused by pressure of a removable prosthesis. A majority of these implants will then already start their lifecycle with the rough surface exposed.

We can find examples of patients with the rough surface exposed without clinical signs of infections or progressive bone loss. However not every patient is susceptible for peri-implant disease or has a good general health with momentarily an

\section{Results by year}

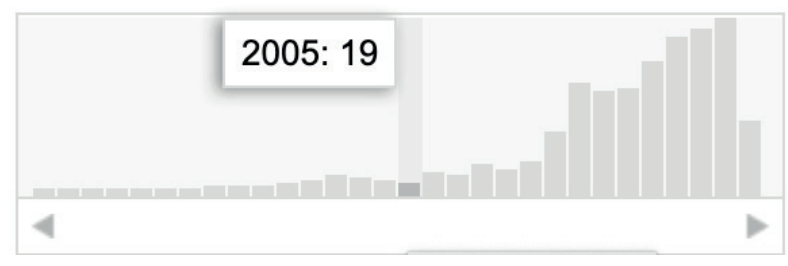

Results by year

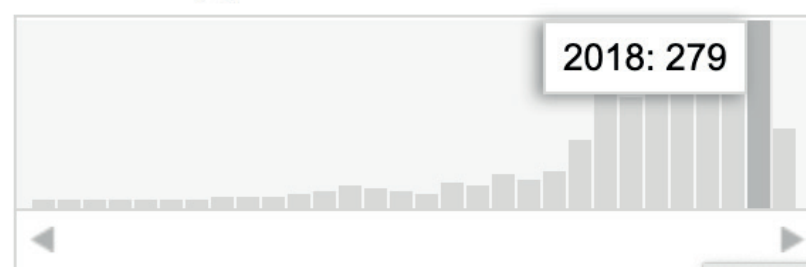

Figure 1. The growing amount of yearly publications of peri-implantitis 
adequate level of oral hygiene. In a >20 year follow-up study by Renvert. ${ }^{2}$ It was found that after these 20 years the oral hygiene of the aging patients became poor. Such changes might change the equilibrium into a progressive and hard to control periimplant disease. We know that with the modern rough implants peri-implantitis is non-linear and also has a rapidly accelerating pattern. ${ }^{3}$

In my experience working in a clinic where we have patients treated over 35 years ago with smooth Bränemark implants and thousands of full (moderate) rough implants of different

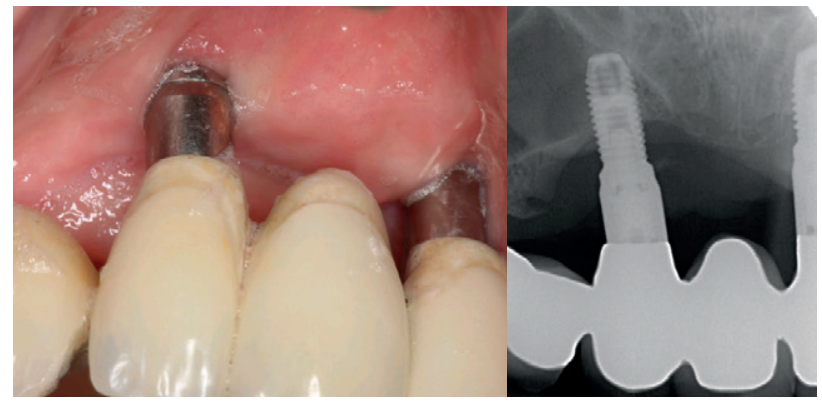

Figure 2. Smooth Bränemark implants placed $>25$ years ago. Resorption of the crista iliac crest bone graft, bone loss around 50\% and lack of keratinized mucosa. Despite these factors a long-term stable result without any signs of peri-implant infection
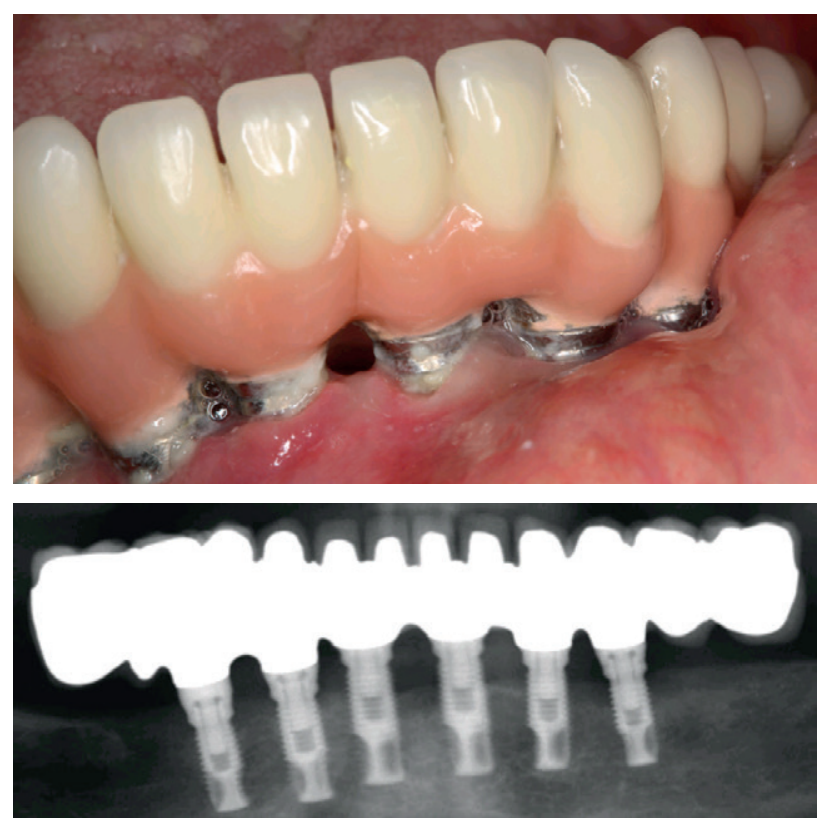

Figure 3. Smooth Bränemark implants placed $>30$ years ago. Patient is momentarily 92 years old and not able to brush anymore. Despite these factors only a mild peri-implant infection with a linear small progressive pattern of bone loss major brands we found some guidance (Figure 2) There's no evidence that a smooth surface will prevent peri-implant infections but the aggressiveness and the predictability to treat the disease differs significantly. In the above mentioned Renvert study it was found that over 20 years in the aging patient group peri-implantitis occurred in $26,7 \%$ of the cases. The general assumption at the moment is that peri-implantitis on patient level occurs around 20\%. The caveat is that this is all based on much shorter observation periods. An increase in occurrence of peri-implantitis with elderly patients with a poorer oral hygiene and all other co-factors is a logical finding. The most interesting conclusion from the study was however that although there was this relatively high percentage of peri-implantitis it didn't lead to major problems, only a few smooth implants failed. However, what would be the outcome of such a long-term study when (moderate) rough implants where used. In such cases implants can lose all the bone within a short period of time due to aggressive non-linear and difficult to treat peri-implantitis (Figure 3,4).
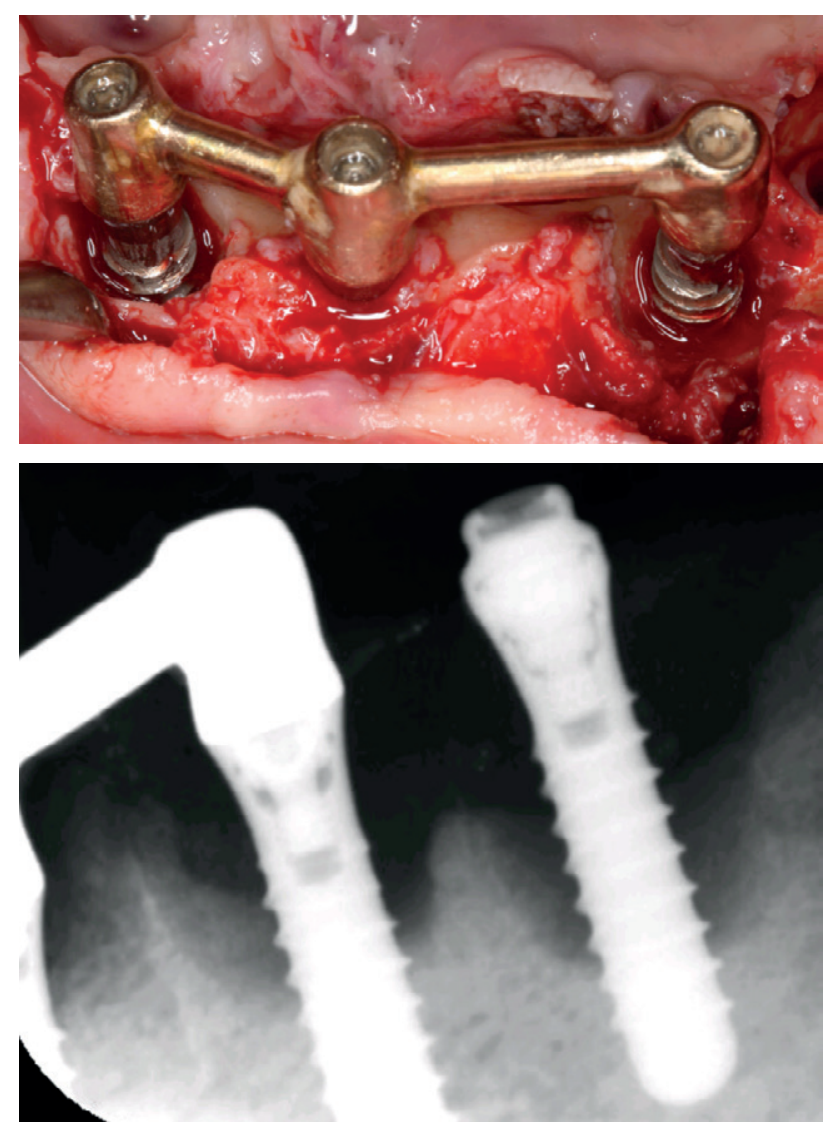

Figure 4. Aggressive peri-implantitis with a non-linear pattern on rough tissue level implants 12 years after placement 
In the literature hints can be found why there might be such differences between rough and smooth surfaces. An in vivo study by Quirynen ${ }^{4}$ tested smooth and rough abutments and found that rough abutments subgingivally harbored 25 times more bacteria than the smooth abutments. There is also an experiment by Berghlund ${ }^{5}$ in Beagle dogs where the progression of periimplantitis around implants with different surface roughness was investigated. It was found that after a 4-month period of ligature induced peri-implantitis the progression of bone loss and the size of the inflammatory lesions were significantly larger in rough than in smooth implants. Such behavior was also found in humans in a recent 3-year follow-up randomized clinical trial by Carcuac. ${ }^{6}$ A total of 100 patients with advanced peri-implantitis were treated. Implant surface characteristics had a significant impact on the 3-year outcomes, in favor of implants with smooth surfaces. $92 \%$ of the smooth implants showed after treatment no further bone loss compared to $62 \%$ of the rough implants. In our experience peri-implantitis on smooth implants can even often be treated without an open flap as therapy. Debridement of the granulation tissue and air polishing heals already a large part of peri-implant infections on smooth implants (Figure $5 \mathrm{a}, \mathrm{b}$ ).

We shouldn't neglect that there are reasons why almost all clinicians 15 years ago shifted from smooth surfaces to rough surfaces. Mainly because of the higher early failure rate of smooth implants and longer healing periods. The implant surface became a huge marketing tool for an implant brand and many studies were conducted to proof prove the superiority of different rough surfaces over the standard smooth (machined) surface. Looking closely at the research it can be seen that these are mostly based on short time ( $<5$ year) studies and that the failure rate of smooth surfaces only differs in type IV bone and in immediate or early loading situations. Also, a theory was postulated that bone would settle on the border of the smooth and the rough surface. This was however based on a short-term animal study and in contradiction with long-term studies where smooth Bränemark implants scored similar results for bone levels as rough microthreaded Astra Tech implants. ${ }^{7}$

In contrary suggestions in the literature can be found for a more stable long-term result with smooth surfaces. A meta-analysis of Doornewaard ${ }^{8}$ showed after 5 years already a less average bone loss around the smoother surfaces. Patients do want short and fast treatments, but they expect their implants to be durable for more than 20 years too. Likely when they have to choose between immediate function or a long-term result, the latter will prevail. A hybrid surface implant which has both advantages, a low early failure rate and short healing periods with long-term stability due to good integration of the mucosa, will be the trend in the coming decade.

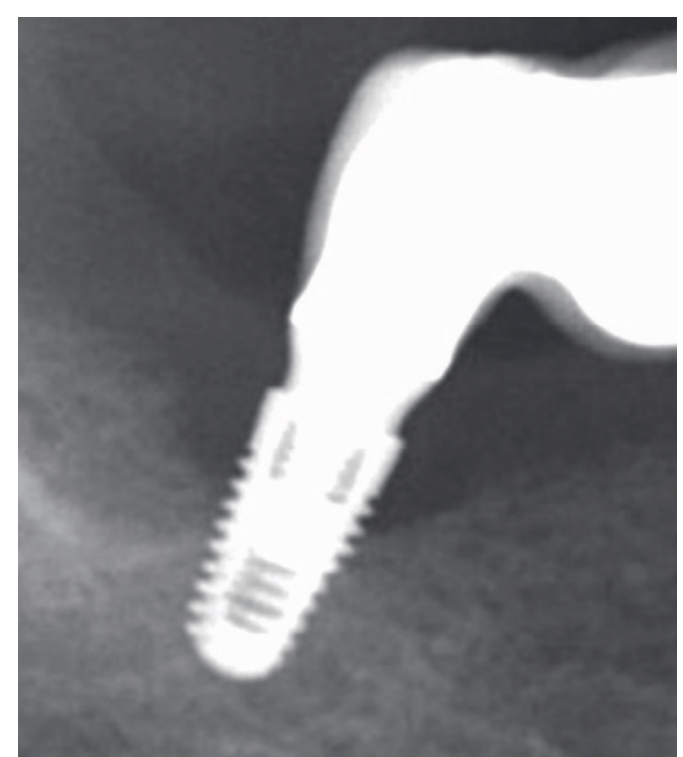

Figure 5a. Early onset peri-implantitis on a hybrid (smooth) implant caused by contact of the removable prosthesis during the initial phase

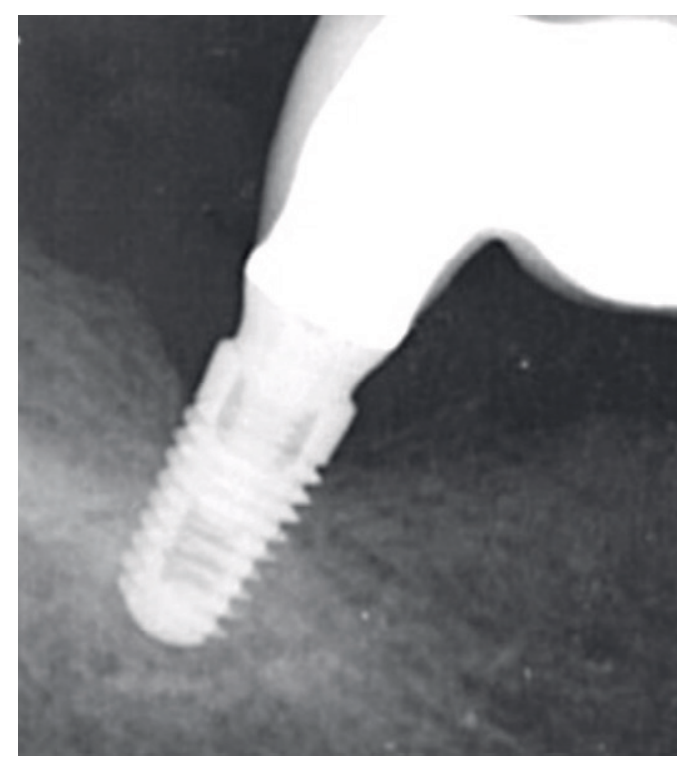

Figure 5b. After a straightforward closed flap treatment with removing the granulation tissue and air polishing the smooth surface there was 6 months later healing of the infection and spontaneous regeneration of the bony defect 
The last misunderstanding is that the rough implant necks gave us the esthetic results we achieve now. The huge leap forward we made in implant dentistry has been driven by the internal connections, immediate implant placement, new mucogingival surgical techniques, zirconia abutments together with the digital (planning) workflow. It's time to forget it's all about rough surfaces and microgrooves (Figure 6a,b,c,d,e,f,g,h).
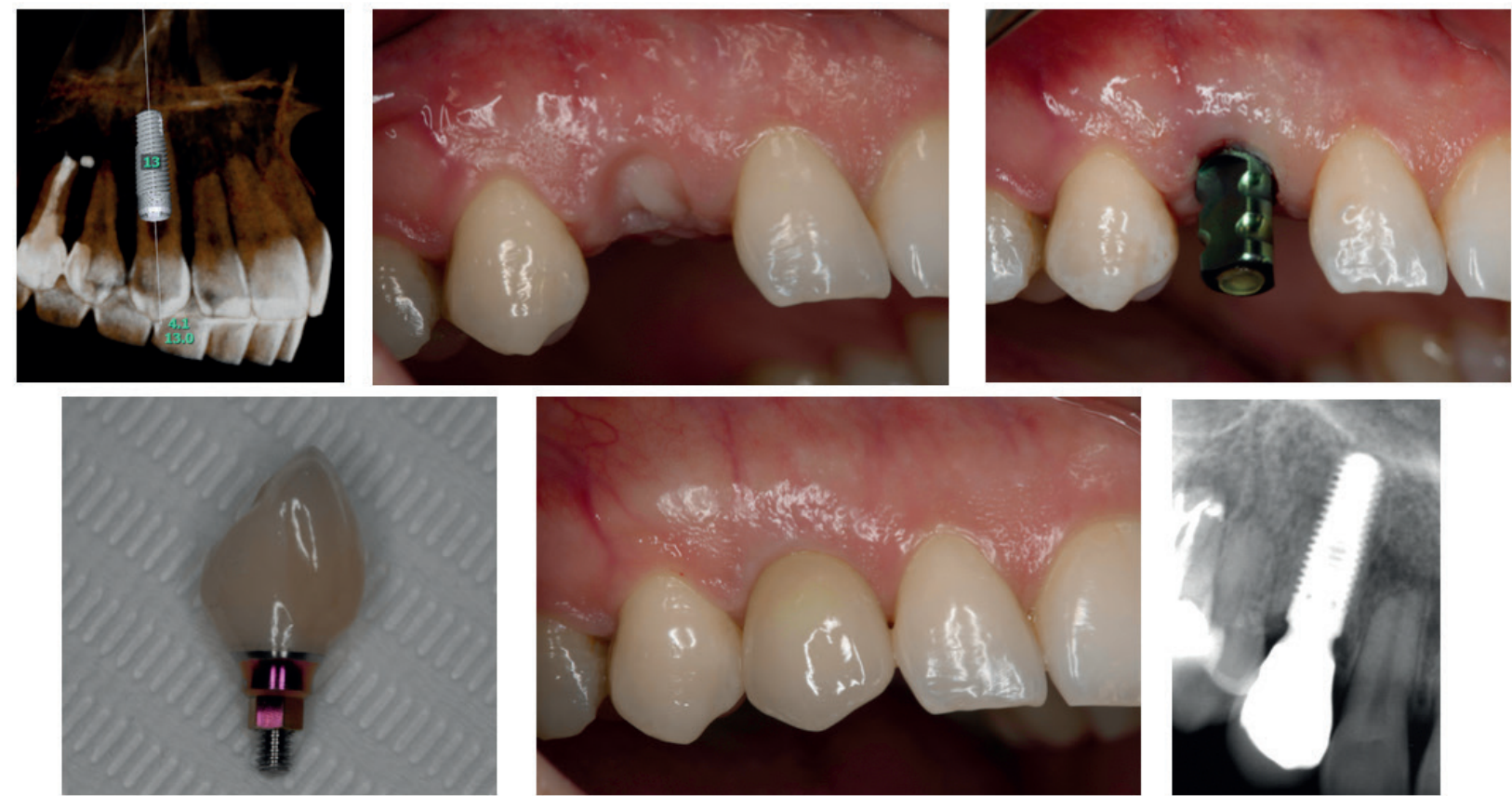

Figure 6a. Immediate replacement of tooth \#13 due to external resorption with a hybrid surface implant Legacy P 4.2x16 mm. X-ray 1 year after crown placement
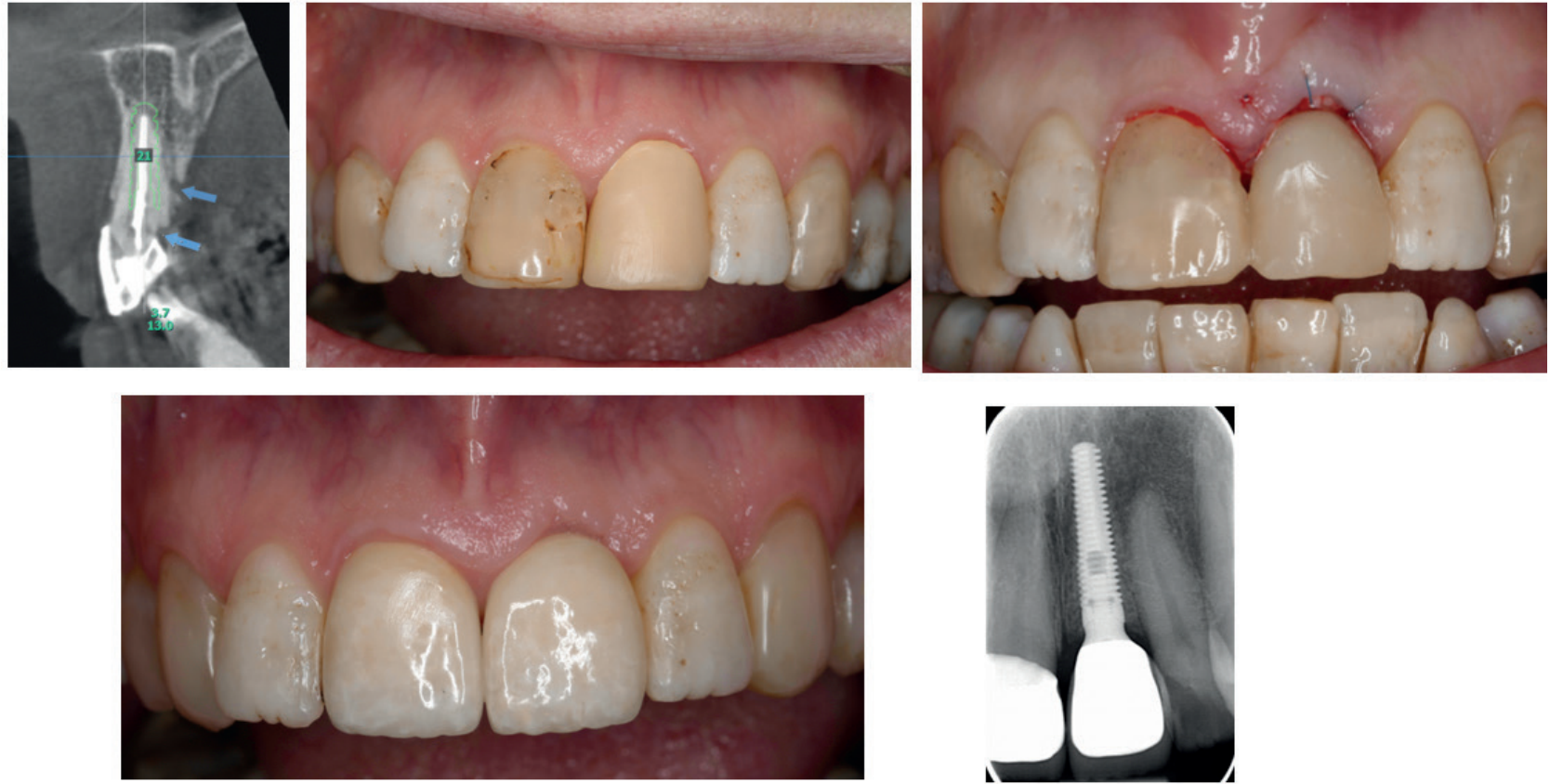

Figure 6b. Immediate replacement of tooth \#21 due to external resorption with a hybrid LegacyP 3,7×16, mm implant 

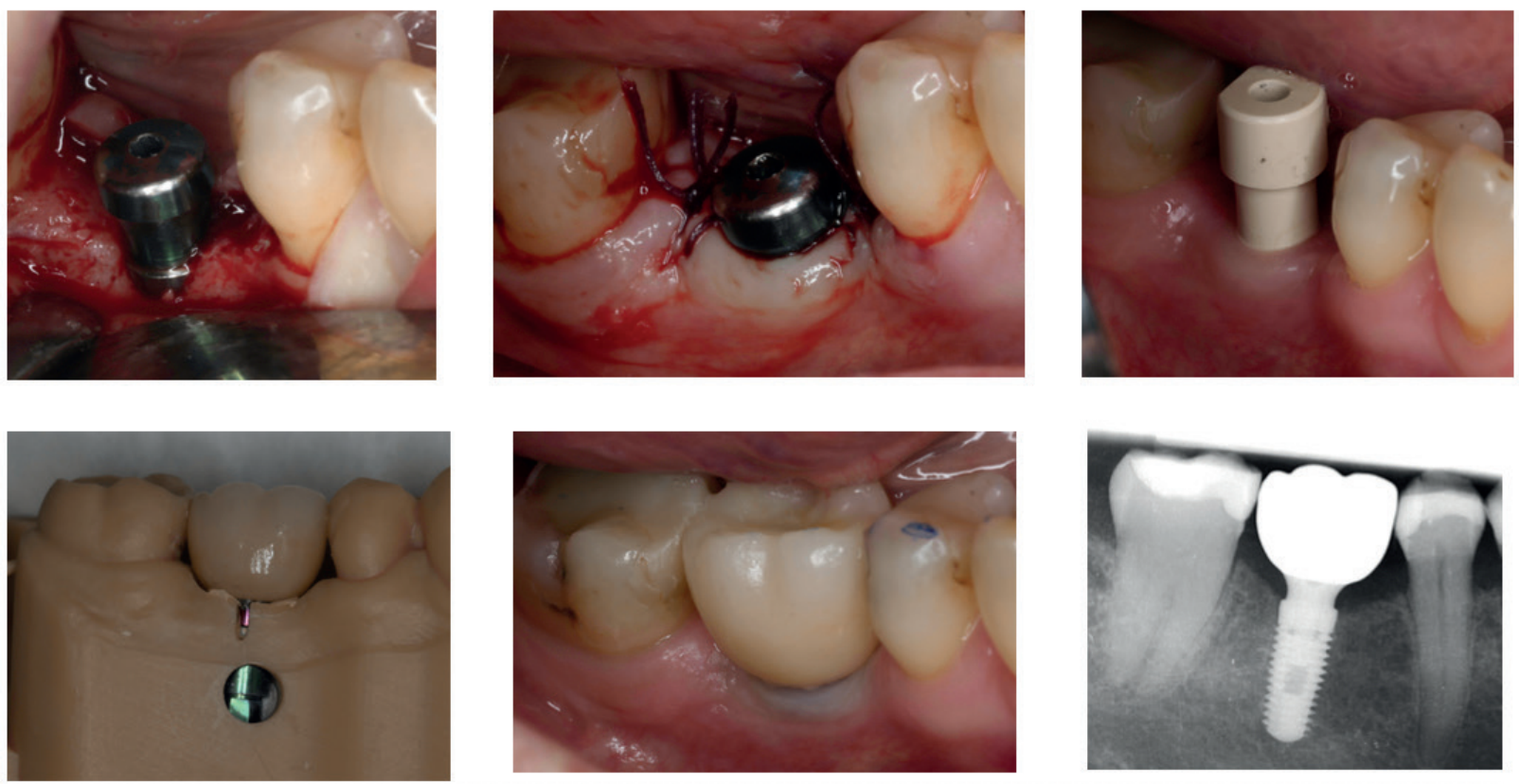

Figure 6c. Implant tooth \#46. Legacy hybrid implant 4.2x10 mm. Intra-oral impression 3Shape and 3D printed model formlabs2. Une of the biggest advantages is the fact that the smooth coronal part can be left exposed during surgery avoiding Guided Bone Regeneration procedures

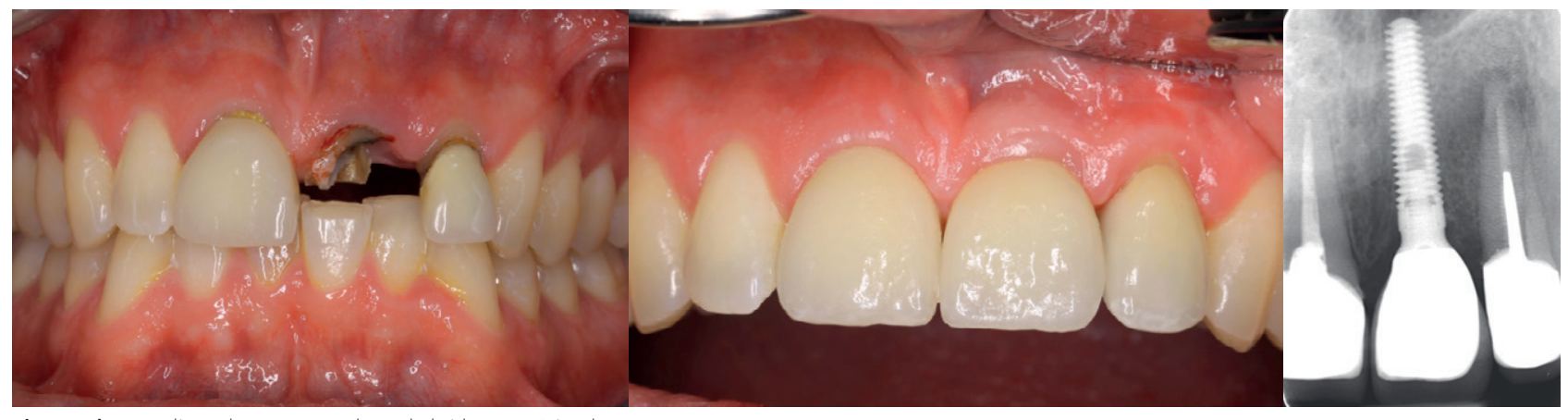

Figure 6d. Immediate placement tooth \#21 hybrid Legacy P implant 3,7×16mm

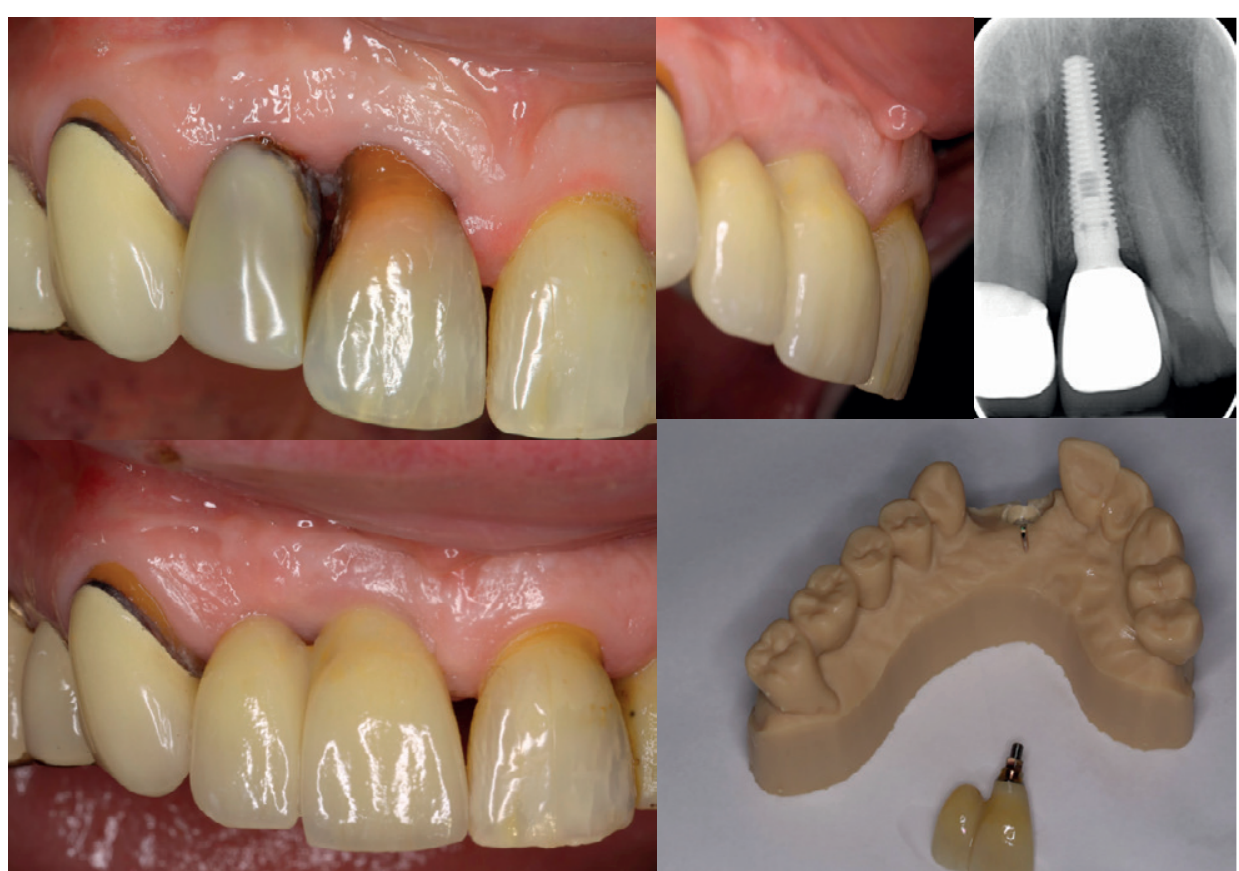

Figure 6e. Replacement of failing teeth \#12\#11 with hybrid Legacy P implant 3,7x13mm and cantilever\#12 


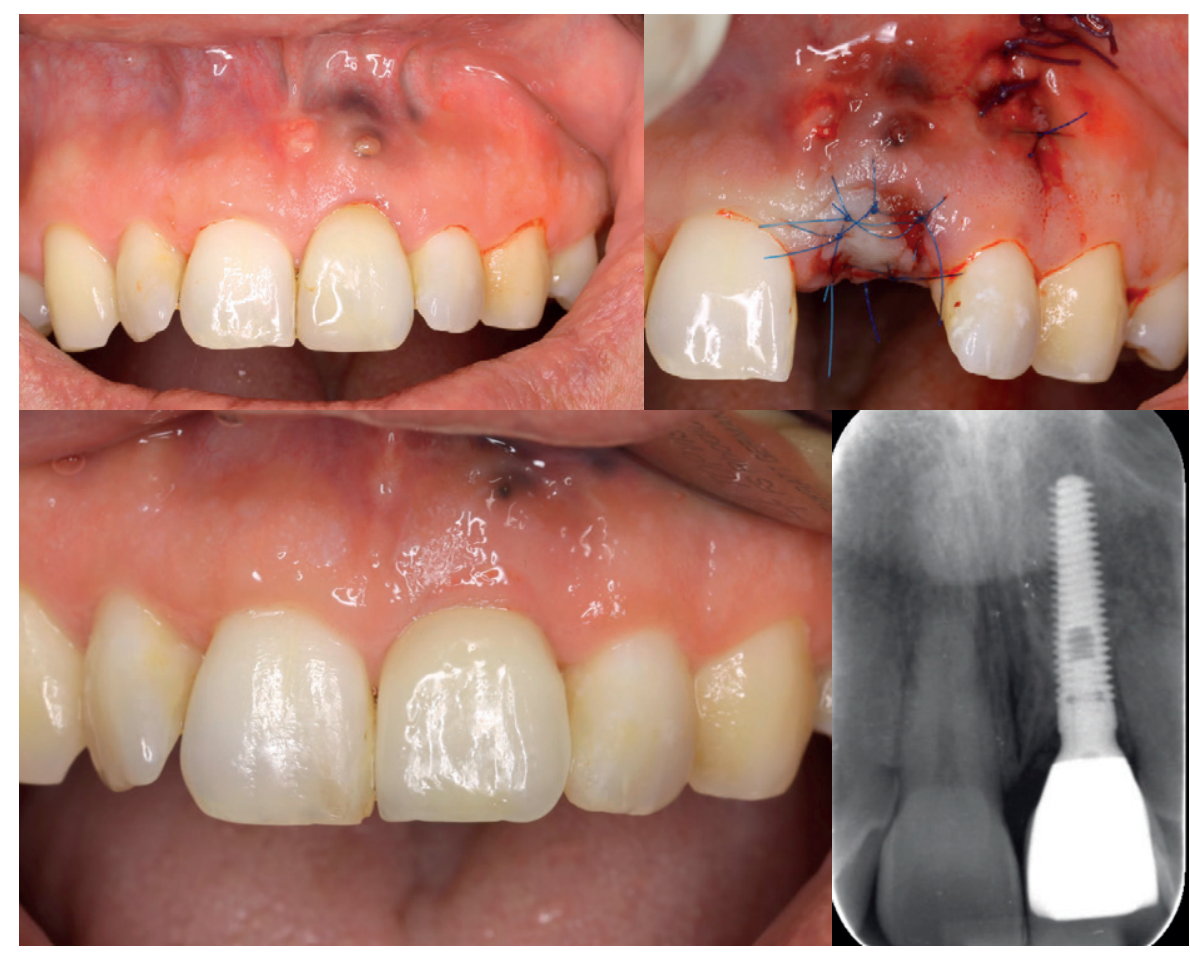

Figure 6f. Immediate placement of tooth \#21

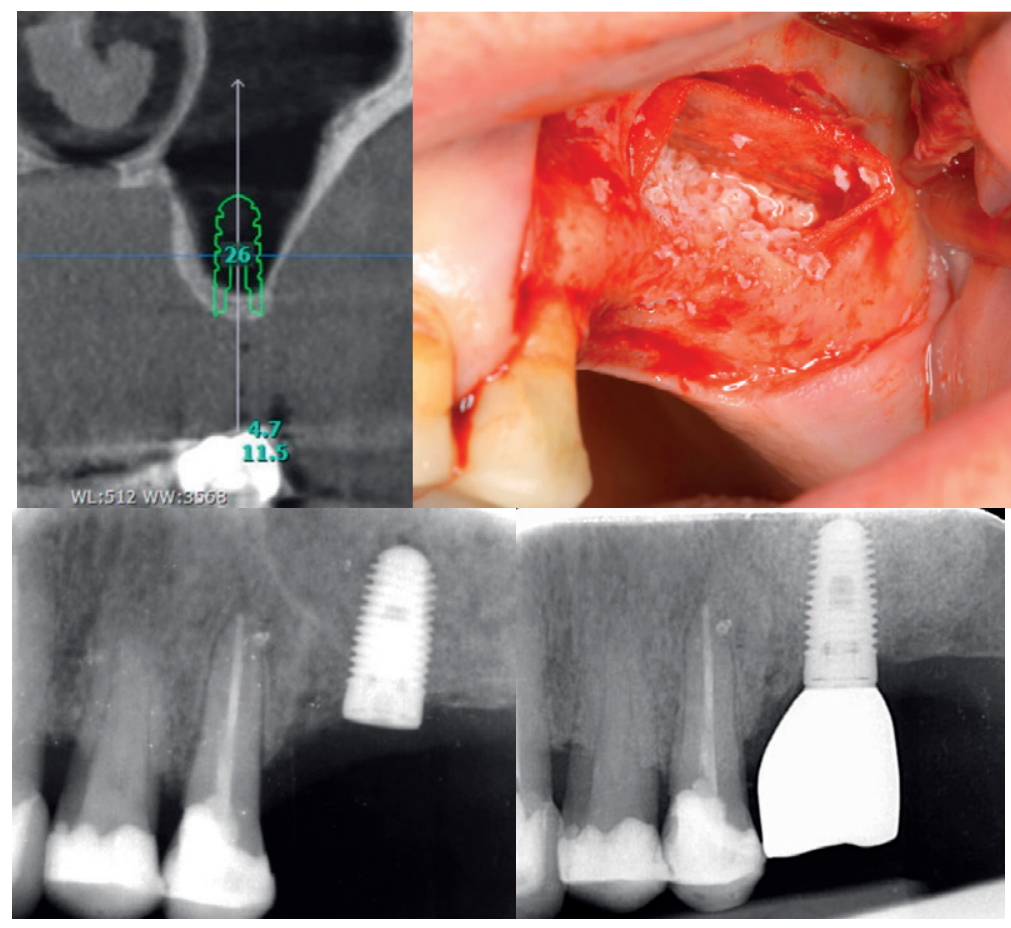

Figure 6g. Lateral sinus augmentation \#26 with Legacy P hybrid implant 4.7×10mm

\section{REFERENCES}

1. Effectiveness of implant therapy analyzed in a Swedish population: early and late implant loss. J Dent Res. 2015 Mar;94(3 Suppl):44S-51S.

2. Occurrence of cases with peri-implant mucositis or peri-implantitis in a 21-26 years follow-up study. J Clin Periodontol. 2018 Feb;45(2):233-240.

3. Peri-implantitis - onset and pattern of progression J Clin Periodontol. 2016 Apr;43(4):383-8.

4. An in vivo study of the influence of the surface roughness of implants on the microbiology of supra- and subgingival plaque. J Dent Res. 1993 Sep;72(9):1304-9.

5. Experimental periodontitis and peri-implantitis in dogs. Clin Oral Implants Res. 2013 Apr;24(4):363-71.

6. Surgical treatment of peri-implantitis: 3-year results from a randomized controlled clinical trial. J Clin Periodontol. 2017 Dec;44(12):1294-1303.

7. The incidence of peri-implantitis for two different implant systems over a period of thirteen years. J Clin Periodontol. 2012 Dec;39(12).

8. Long-Term Effect of Surface Roughness and Patients' Factors on Crestal Bone Loss at Dental Implants. A Systematic Review and Meta-Analysis. Clin Implant Dent Relat Res. 2017 Apr;19(2):372-399. 